\title{
Urinary Microbiome and Psychological Factors in Women with Overactive Bladder
}

\author{
Peng $W^{1 * \dagger}$, Yang Chen ${ }^{1 \dagger}$, Jie Zhao ${ }^{2 \dagger}$, Guihao Zhang ${ }^{1}$, Jiawei Chen ${ }^{1}$, Junpeng Wang ${ }^{1}$ and \\ Huijian Zhang ${ }^{1}$ \\ ${ }^{1}$ Department of Urology, Nanfang Hospital, Southern Medical University, Guangzhou, China, ${ }^{2}$ School of Pharmaceutical \\ Sciences, Southern Medical University, Guangzhou, China
}

OPEN ACCESS

Edited by:

D. Scott Merrell, Uniformed Services University,

United States

Reviewed by:

Alan J. Wolfe

Loyola University Chicago,

United States

Lisa Karstens,

Oregon Health \& Science University,

United States

*Correspondence:

Peng Wu

doctorwupeng@gmail.com

${ }^{\dagger}$ These authors have contributed equally to this work.

Received: 30 July 2017 Accepted: 13 November 2017 Published: 27 November 2017

Citation:

Wu P, Chen Y, Zhao J, Zhang G,

Chen J, Wang J and Zhang H (2017)

Urinary Microbiome and Psychological

Factors in Women with Overactive

Bladder.

Front. Cell. Infect. Microbiol. 7:488,

doi: 10.3389/fcimb.2017.00488
Objectives: Emerging evidence indicates that alterations to the urinary microbiome are related to lower urinary tract symptoms. Overactive bladder $(\mathrm{OAB})$ is a common disorder with complex etiologies and usually accompanied by psychological diseases. More information concerning the urinary microbiome and psychological factors in $O A B$ is required. The aim of this study was to characterize the female urinary microbiome associated with $\mathrm{OAB}$ and investigate the relationships between urinary microbiome and psychological factors.

Methods: Thirty women with $\mathrm{OAB}$ and 25 asymptomatic controls were recruited and asked to finish the Overactive Bladder Symptom Score, Self-Rating Anxiety Scale and Self-Rating Depression Scale. Urine specimens were collected by transurethral catheterization and processed for 16S rRNA gene sequencing using Illumina MiSeq. Sequencing reads were processed using QIIME. LEfSe revealed significant differences in bacterial genera between controls and OAB patients. The relationships between the diversity of the urinary microbiome and psychological scores were identified by Pearson's correlation coefficient.

Results: We found that bacterial diversity (Simpson index) and richness (Chao1) were lower in OAB samples compared to controls $(P$ both $=0.038)$. OAB and control bacterial communities were significantly different (based on weighted UniFrac distance metric, $R$ $=0.064, P=0.037$ ). LEfSe demonstrated that 7 genera were increased (e.g., Proteus and Aerococcus) and 13 were reduced (e.g., Lactobacillus and Prevotella) in OAB group compared to controls. There were negative correlations between scores on Self-Rating Depression Scale and both richness (Chao1, $r=-0.458, P=0.011$ ) and diversity (Shannon index, $r=-0.516, P=0.003$ ) of urinary microbiome in $\mathrm{OAB}$ group. Some bacterial genera of OAB women with anxiety or depression were significantly different from those without.

Conclusions: The aberrant urinary microbiome with decreased diversity and richness may have strong implications in pathogenesis and treatment of OAB. Psychological conditions were correlated with characteristics of urinary microbiome in women with $\mathrm{OAB}$. Further research is needed to understand the connection between central nervous system and urinary microbiome.

Keywords: bacteria, depression, overactive bladder, psychology, urinary microbiome 


\section{INTRODUCTION}

The microbiome is increasingly considered as an essential factor in human health and disease (Young, 2017). Following the completion of the National Institutes of Health Human Microbiome Project, there have been numerous studies identifying and cataloging the microbiome in physiological and pathological states including gastrointestinal diseases, metabolic diseases, infectious diseases, and cancer (Whiteside et al., 2015). The female urinary tract is non-sterile, as bacteria were detected in urines from asymptomatic people using $16 \mathrm{~S}$ rRNA gene sequence analysis and/or an expanded quantitative urine culture (EQUC) (Fouts et al., 2012; Wolfe et al., 2012; Hilt et al., 2014; Pearce et al., 2014). Additionally, studies have shown that standard culture missed $90 \%$ of the bacteria detected by EQUC (Hilt et al., 2014; Pearce et al., 2014). Similar to the influence of microbial communities in other niches, alterations to the urinary microbiome may have an effect on urinary tract disorders. Emerging evidence indicates that shifts in the normal microbiome of the bladder may play an important role in pathophysiology of lower urinary tract symptoms (LUTS) (Siddiqui et al., 2012; Pearce et al., 2014; Nickel et al., 2015; Karstens et al., 2016; Abernethy et al., 2017; Thomas-White et al., 2017).

Overactive bladder $(\mathrm{OAB})$ is a subset of LUTS characterized by urgency, with or without urgency urinary incontinence (UUI), usually with frequency and nocturia, in the absence of urinary tract infection (UTI), or other identifiable causes (Abrams et al., 2002). OAB symptoms affect social, psychological, occupational, domestic, physical, and sexual aspects of life and lead to a heavy cost (Stewart et al., 2003). Some $\mathrm{OAB}$ symptoms are clearly due to neuromuscular issues and muscarinic receptors, but some individuals with $\mathrm{OAB}$ do not respond to anti-muscarinic receptor, botox, or other treatments (Gormley et al., 2015), which suggests that their symptoms might arise from other causes or that the causes are complex. Therefore, it is an urgent need to further investigate the pathogenesis of $\mathrm{OAB}$ and identify new therapeutic target.

A brain-gut-microbiome axis between the brain, the gut, and the gut microbiome has been well identified (Mayer et al., 2014). The urinary microbiome might play similar roles, given the wellknown connection between central nervous system and bladder function (Tadic et al., 2010). OAB patients had higher levels of depression, anxiety and embarrassment than asymptomatic people (Kinsey et al., 2016). Bradley et al. found that female veterans with anxiety symptoms were more likely to have bothersome urgency incontinence and/or frequency symptoms (Bradley et al., 2014). It was reported that OAB patients with depression had more severe urinary incontinence symptoms, greater bother and more impact on quality of life compared to those without depression (Lai et al., 2016). There have been some studies on the urinary microbiome of OAB/UUI (Hilt et al., 2014; Pearce et al., 2014; Karstens et al., 2016; Curtiss et al., 2017; Thomas-White et al., 2017), but no previous study researched the urinary microbiome considering psychological disorders. Our primary purpose was to characterize the female urinary microbiome associated with $\mathrm{OAB}$ in China and investigate relationships between the microbiome and psychological factors.

\section{MATERIALS AND METHODS}

\section{Subject Recruitment and Urine Collection}

Between September 2016 and March 2017, adult patients aged 18 or above, diagnosed with $\mathrm{OAB}$, and asymptomatic controls were recruited into this study at Nanfang Hospital in China. Patients must complain of urinary urgency, with or without urgency incontinence, usually with frequency and nocturia, in the absence of infection or other identifiable causes, in accordance with the 2002 ICS definition of OAB (Abrams et al., 2002). Asymptomatic controls must have no prior diagnosis of $\mathrm{OAB}$ or interstitial cystitis/bladder pain syndrome, no pelvic pain, and no evidence of urinary infection. Subjects with a history of urethral stricture disease, urinary retention, pelvic radiation, tuberculosis cystitis, cyclophosphamide cystitis, genitourinary cancer, urinary stones, neurogenic bladder, a documented positive urine culture in the past 6 weeks, or a post-void residual volume $\geq 150 \mathrm{~mL}$ were not eligible. The Medicine Institutional Review Board of Southern Medical University approved this study, and written informed consents from all participants were obtained. All participants were required to finish Overactive Bladder Symptom Score (OABSS), Self-Rating Anxiety Scale (SAS, standard score $\geq 50$ indicates anxiety) and Self-Rating Depression Scale (SDS, standard score $\geq 53$ indicates depression). Many studies investigated the urinary microbiome based on the collection of mid-stream urine into a sterile container (Siddiqui et al., 2012; Lewis et al., 2013; Thomas-White et al., 2017). However, the collection of mid-stream urine cannot avoid contamination from the distal urethra, vulva, or vagina. Sterile alternatives like transurethral catheter and suprapubic aspirate might be more adequate and elaborate for scientific exploration of the bladder microbiome (Wolfe et al., 2012; Hiergeist and Gessner, 2017). Therefore, $50 \mathrm{ml}$ of urine from each participant was collected through transurethral catheterization in this study. Twenty milliliter of the specimen was tested by standard cultivation to exclude UTI. The rest of specimen $(30 \mathrm{ml})$ was kept at $4^{\circ} \mathrm{C}$, and immediately shifted to laboratory within an hour for centrifugation at $16,000 \mathrm{~g}$ for $10 \mathrm{~min}$. The pellets were stored at $-80^{\circ} \mathrm{C}$ until further processing.

\section{DNA Isolation and 16S rRNA Gene Sequencing}

DNA extraction was performed using the cultured cells protocol supplied with the DNeasy Blood and Tissue Kit (Qiagen, Germany) in a laminar flow hood to avoid contamination. The concentration of extracted DNA was determined using a Nanodrop ND-1000 spectrophotometer (Thermo Electron Corporation, USA). PCR amplification of $16 \mathrm{~S}$ rDNA sequences was performed using primer sets specific for V4 regions. Extraction negative controls (no urine) and PCR negative controls (no template) were included to assess contribution of extraneous DNA from reagents. Final PCR products were purified from unincorporated nucleotides and primers using 
the Qiaquick PCR purification kit (Qiagen, Valencia, USA). Purified samples were normalized to equal DNA concentration and sequenced using the Illumina Miseq sequencer (Illumina, USA). The $16 \mathrm{~S}$ rRNA gene sequences have been submitted to the Short Read Archive (SRA) under accession number SUB2843807.

\section{Statistical Analysis \\ Clinical Data Analysis}

Differences in demographic characteristics between patients and controls were evaluated using Mann-Whitney $U$-test for continuous variables and Pearson's chi-square test for count data. Bivariate correlation analyses were conducted to detect the direction and strength of relations between clinical data and indices of bacterial alpha diversity using Pearson's correlation. Statistical analysis was performed using the Statistical Package for Social Science (SPSS, version 21, USA). For differentially abundant taxa (only taxa with mean relative abundance more than $1 \%$ were tested) between cohorts, Wilcoxon rank sum test was applied, and Benjamini-Hochberg false discovery rate correction was performed in $\mathrm{R}$ (version 3.4.1, stats package). Statistical tests were based on two-tailed probability. The conventional $P<0.05$ was used to assess the statistical significance.

\section{Bioinformatics Analysis}

The wrapper package Quantitative Insights Into Microbial Ecology (QIIME) was applied to process the raw reads to create an operational taxonomic units (OTUs) table (Caporaso et al., 2010). Sequences were clustered into individual OTUs at a default similarity level of $97 \%$ using an open reference picking strategy with Uclust, and subsequently, chimera detection was performed using the UCHIME method (Edgar, 2010). A single representative sequence from each clustered OTU was used to align to the Greengenes database using Ribosomal Database Project Classifier (Wang et al., 2007).

Alpha diversity was evaluated by calculating four indices using QIIME, including the Observed species, Chao1, Shannon, and Simpson indices. Pielou index is an indicator of species evenness generated in $\mathrm{R}$ (version 3.4.1, vegan package), whereas Chaol and Observed species represent bacterial richness. Shannon and Simpson indices are quantitative measures of bacterial diversity reflecting both species richness and evenness. The difference of alpha diversity was evaluated by Kruskal-Wallis test. To compare microbial composition between samples, betadiversity was measured by calculating the Bray Curtis, weighted UniFrac and unweighted UniFrac distances. Principal coordinate analysis (PCoA) was applied on the distance matrices to generate three-dimensional plots in QIIME. The analysis of similarities (ANOSIM) of variance of Bray Curtis, weighted UniFrac, and unweighted UniFrac distances within $\mathrm{R}$ software was used to calculate $P$-values and test for significant differences in betadiversity between groups.

To identify significantly different bacteria as biomarkers between groups at genus level, taxa summaries were reformatted and inputted into Linear discriminant analysis effect size (LEfSe) via the Huttenhower Lab Galaxy Server (Segata et al., 2011). In the settings of LEfSe, the Kruskal-Wallis sum-rank test $(\alpha=0.05)$ was used to detect taxa with significant differential abundance at first; as a second step, biological consistency was then investigated with a set of pairwise tests among subclasses using the Wilcoxon rank-sum test; finally, linear discriminant analysis (LDA) was used to estimate the effect size of differentially abundant genera (Segata et al., 2011). The threshold on the logarithmic LDA score for discriminative features was 2.5 .

\section{RESULTS}

\section{Participant Demographic Characteristics and Clinical Symptoms}

Thirty women with $\mathrm{OAB}$ and 30 asymptomatic controls were recruited. However, five samples in the control group were excluded because three of them contained a few sequencing reads and two were suspected to contain contaminants. There was no significant difference in the demographic characteristics between two cohorts (Table 1, Supplementary Table 1), except the previous anticholinergic use (OAB 37\%, control 0\%, $P=$ $0.001)$. The median age of patients was 27.5 years and of controls was 26.0 years $(P=0.258)$. Most of these participants were currently married (OAB 70\%, control 75\%) and premenopausal

TABLE 1 | Comparisons of demographic characteristics and scale scores between OAB patients and asymptomatic controls.

\begin{tabular}{|c|c|c|c|}
\hline Characteristic & $\begin{array}{l}\text { Patients with } \\
\text { OAB }(n=30)\end{array}$ & $\begin{array}{l}\text { Asymptomatic } \\
\text { controls }(n=25)\end{array}$ & $P$-value \\
\hline Age (y) & $27.5(26.0,35.3)$ & $26.0(23.0,47.5)$ & 0.258 \\
\hline BMl & $20.6(18.6,22.2)$ & $20.0(19.0,21.8)$ & 0.754 \\
\hline Currently married & $21(70)$ & $19(75)$ & 0.619 \\
\hline Ever pregnant & $19(63)$ & $14(56)$ & 0.580 \\
\hline Premenopausal & $26(87)$ & $20(80)$ & 0.506 \\
\hline Estrogen treatment & $3(10)$ & 0 & 0.104 \\
\hline DM & $2(6)$ & 0 & 0.188 \\
\hline $\mathrm{HP}$ & $1(3)$ & 0 & 0.357 \\
\hline Previous anticholinergic use & $11(37)$ & 0 & 0.001 \\
\hline Gynecologic diseases & $3(10)$ & $5(20)$ & 0.295 \\
\hline Pelvic surgery & $2(6)$ & $1(4)$ & 0.665 \\
\hline OABSS & $8.0(6.0,10.0)$ & $1.0(0,2.5)$ & $<0.001$ \\
\hline Q1. Daytime frequency & $2.0(1.0,2.0)$ & $0(0,1.0)$ & $<0.001$ \\
\hline Q2. Nighttime frequency & $2.0(2.0,3.0)$ & $0(0,1.0)$ & $<0.001$ \\
\hline Q3. Urgency & $4.0(2.8,5.0)$ & $0(0,1.0)$ & $<0.001$ \\
\hline Q4. Urgency incontinence & $0(0,0)$ & $0(0,0)$ & 0.572 \\
\hline SDS & $48.5(45.0,58.8)$ & $40.0(30.5,48.5)$ & $<0.001$ \\
\hline Mild or moderate depression & $14(47)$ & $5(20)$ & 0.038 \\
\hline Severe depression & 0 & 0 & ns \\
\hline SAS & $48.0(46.0,59.3)$ & $41.0(33.0,45.5)$ & $<0.001$ \\
\hline Mild or moderate anxiety & $11(37)$ & $3(12)$ & 0.037 \\
\hline Severe anxiety & $1(3)$ & 0 & 0.357 \\
\hline
\end{tabular}

Data are reported as median (interquartile range) for continuous variables or $n$ (\%) for counts. BMI, Body mass index; DM, diabetes mellitus; HP, hypertension; OABSS, Overactive Bladder Symptom Score; SDS, Self-Rating Depression Scale; SAS, SelfRating Anxiety Scale; and ns, not significant (based on $P<0.05$ ). Standard scores on SAS $\geq 50$ indicate the presence of anxiety; standard scores on $S D S \geq 53$ indicate depression. 
(OAB 87\%, control 80\%). For the OABSS, the total score and some sub-scores (daytime frequency, nighttime frequency and urgency) were significantly higher in the $\mathrm{OAB}$ group than in the controls (Table 1, $P$ all $<0.001)$. Twelve $(40 \%)$ of the OAB group had anxiety (median score 48.0 ), one of which reported severe anxiety, and 14 (47\%) suffered from mild or moderate depression (median score 48.5$)$. In the control group, 3 (12\%) had mild or moderate anxiety (median score 41.0$)$, and 5 (20\%) suffered from mild or moderate depression (median score 40.0). The prevalence of anxiety and the prevalence of depression in the $\mathrm{OAB}$ group were both markedly higher than in the control group $(P<0.05)$.

\section{Sequencing Data, Alpha, and Beta Diversity}

We obtained 6,605,388 sequences from the 55 samples. The median number of reads in OAB group was 110,250 , and in the control group was 102,125 (Table 2, $P=0.685$ ). The reads were classified into 39,905 OTUs that were used for downstream analyses. No difference was found in the number of OTUs between the two cohorts (Table 2, $P=0.073$ ).

The indices of bacterial alpha diversity were listed in Table 2 and Supplementary Table 2. There were no differences in the Observed species (Figure 1A) and Shannon index (Figure 1C) between $\mathrm{OAB}$ and controls. The Chaol was significantly lower in the $\mathrm{OAB}$ cohort than in the control cohort $(P=0.038$; Figure 1B). The Simpson index illustrated the bacterial diversity was markedly lower in the OAB patients $(P=0.038$; Figure 1D). However, the Pielou index indicated that there was no significant difference in bacterial evenness between the two groups $(P=$ 0.205; Figure 1E).

The PCoA was performed to measure the extent of the similarity of microbial communities in the two cohorts based on unweighted UniFrac (Figure 1F), weighted UniFrac (Figure 1G) and Bray Curtis (Figure $\mathbf{1 H}$ ) distance metrics. Some OAB patients clustered away from controls in the PCoA plot and hierarchical clustering based on weighted UniFrac distance metric (Figures 1G, 2A), but there were overlapping regions where other $\mathrm{OAB}$ women and controls were located. MannWhitney $U$-test was applied to analyze variables between $\mathrm{OAB}$ patients clustered away from controls and other patients mixed with controls. We found that scores on OABSS, SAS, and SDS in $\mathrm{OAB}$ patients clustered away from controls (cluster 1 and cluster 3 in Figure 2A) were higher than in patients mixed with controls (cluster 2 in Figure 2A), although without statistical significance (Supplementary Table 3). Specifically, ANOSIM revealed that there were significant differences in bacterial communities between $\mathrm{OAB}$ and control groups using the BrayCurtis distance metric (statistic $R=0.055, P=0.038$, number of permutations $=999$ ) and weighted UniFrac distance metric (statistic $R=0.064, P=0.037$, number of permutations $=999$ ). However, this clustering was not significant when the analysis was based on unweighted UniFrac distance metric (statistic $R=$ $0.023, P=0.068$, number of permutations $=999$ ).

\section{Relative Abundances of Urinary Bacteria in Control and OAB Samples}

The genera compositions of all samples were also demonstrated in Figure 2A and Supplementary Table 4. The control group had a higher degree of diversity than the OAB group. Half of control samples were dominated by Lactobacillus. However, the microbiome of $\mathrm{OAB}$ group displayed greater variability, and could be divided into three clusters. The mean relative abundances of various bacterial phyla and families in each group were listed in Table 2 and summarized in Figures 2B,C. The most frequently detected phylum was Firmicutes $(42.7 \%$ $\mathrm{OAB}, 56.4 \%$ control), followed by Proteobacteria $(25.0 \% \mathrm{OAB}$, $14.3 \%$ control), Actinobacteria (19.8\% OAB, 6.8\% control, $P=$ 0.048 ) and Bacteroidetes ( $8.1 \% \mathrm{OAB}, 14.3 \%$ control). At the family level, Bifidobacteriaceae $(P=0.012)$ was significantly more abundant in the $\mathrm{OAB}$ group. Venn diagrams demonstrated that 375 families (Figure 3A) and 689 genera (Figure 3B) were shared between the two cohorts. The bacterial compositions of all samples at different levels were shown in Supplementary Figure 1.

\section{Specific Genera Associated with OAB}

The LEfSe algorithm was utilized to identify the specific bacteria genera associated with $\mathrm{OAB}$ (Figure 4). By specifying control and $\mathrm{OAB}$ as distinct classes, the LEfSe revealed that 13 genera were underrepresented in the OAB group, including Prevotella, Dialister, Fusobacterium, Jonquetella, Campylobacter, Finegoldia, Anaerococcus, Lactobacillu, Pyramidobacter, Ureaplasma, Enterococcus, Novosphingobium, and Lactococcus. In contrast, there were seven genera overrepresented in OAB group, including Sneathia, Staphylococcus, Proteus, Helcococcus, Gemella, Mycoplasma, and Aerococcus.

\section{Urinary Microbiome and Psychological Symptoms in OAB Patients}

We compared several variables between anxiety group and non-anxiety group, and depression group and non-depression group in $\mathrm{OAB}$ patients, respectively. We found that $\mathrm{OAB}$ patients with anxiety or depression reported significantly higher scores on OABSS (Table 3, $P$ all $<0.05$ ). The alpha diversity indices (Observed species, Chaol, Simpson, and Shannon indices) were markedly lower in depression cases than in non-depression cases (Table 3, $P$ all $<0.01$ ). Higher score on SDS inversely correlated with both Shannon index $(r=-0.516$, $P=0.003$, Figure 5A) and Chaol $(r=-0.458, P=0.011$, Figure 5B). LEfSe algorithm was also applied to identify the specific genera that were differentially represented in different psychological status in $\mathrm{OAB}$ patients. Based on LEfSe resluts, Burkholderia, Cryocola, Agrobacterium, Methanobrevibacter, Collinsella, and Psychrobacter were relatively more abundant in the non-anxiety cohort compared to the anxiety cohort (Figure 5C). In contrast, Sneathia, Porphyromonas, Gallicola, Leptolyngbya, Alicyclobacillus, Helcococcus, and Actinobaculum were significantly enriched in the $\mathrm{OAB}$ patients with anxiety (Figure 5C). Compared to the depression cohort, more Actinobaculum, Pedobacter, WAL_1855D, and Clostridium were found in the non-depression cohort (Figure 5D).

\section{DISCUSSION}

In the present study, we characterized the urinary microbiomes of female $\mathrm{OAB}$ patients and asymptomatic women using high throughput sequencing of the bacterial $16 \mathrm{~S}$ rRNA gene. The bacterial diversity and richness were decreased in the OAB 
TABLE 2 | Comparisons of parameters of microbiome and mean relative abundances of bacteria (as percent reported by phyla: family) in OAB urine and control urine.

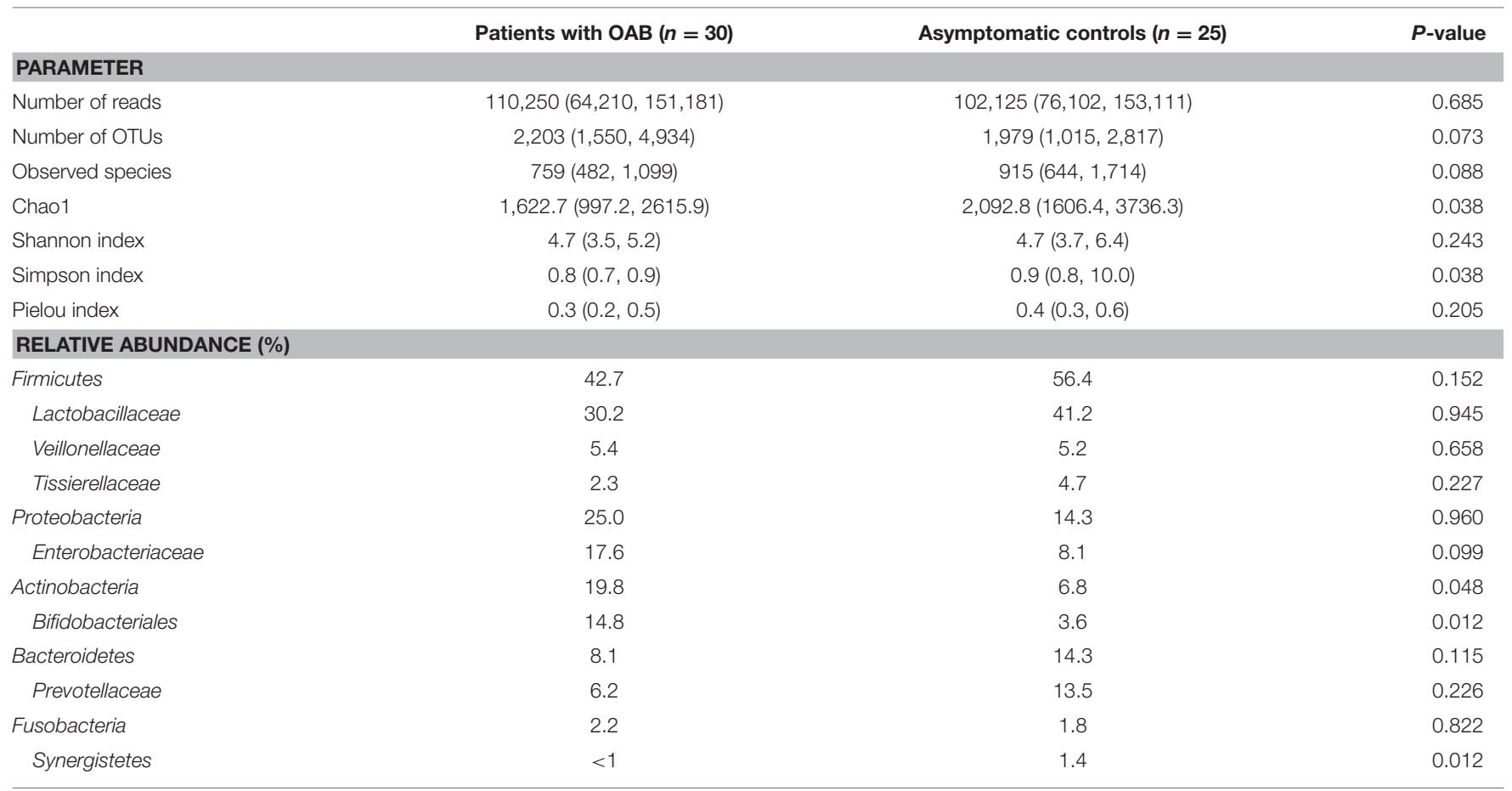

Data are reported as median (interquartile range) for parameters. OAB, Overactive bladder; OTUs, operational taxonomic units.
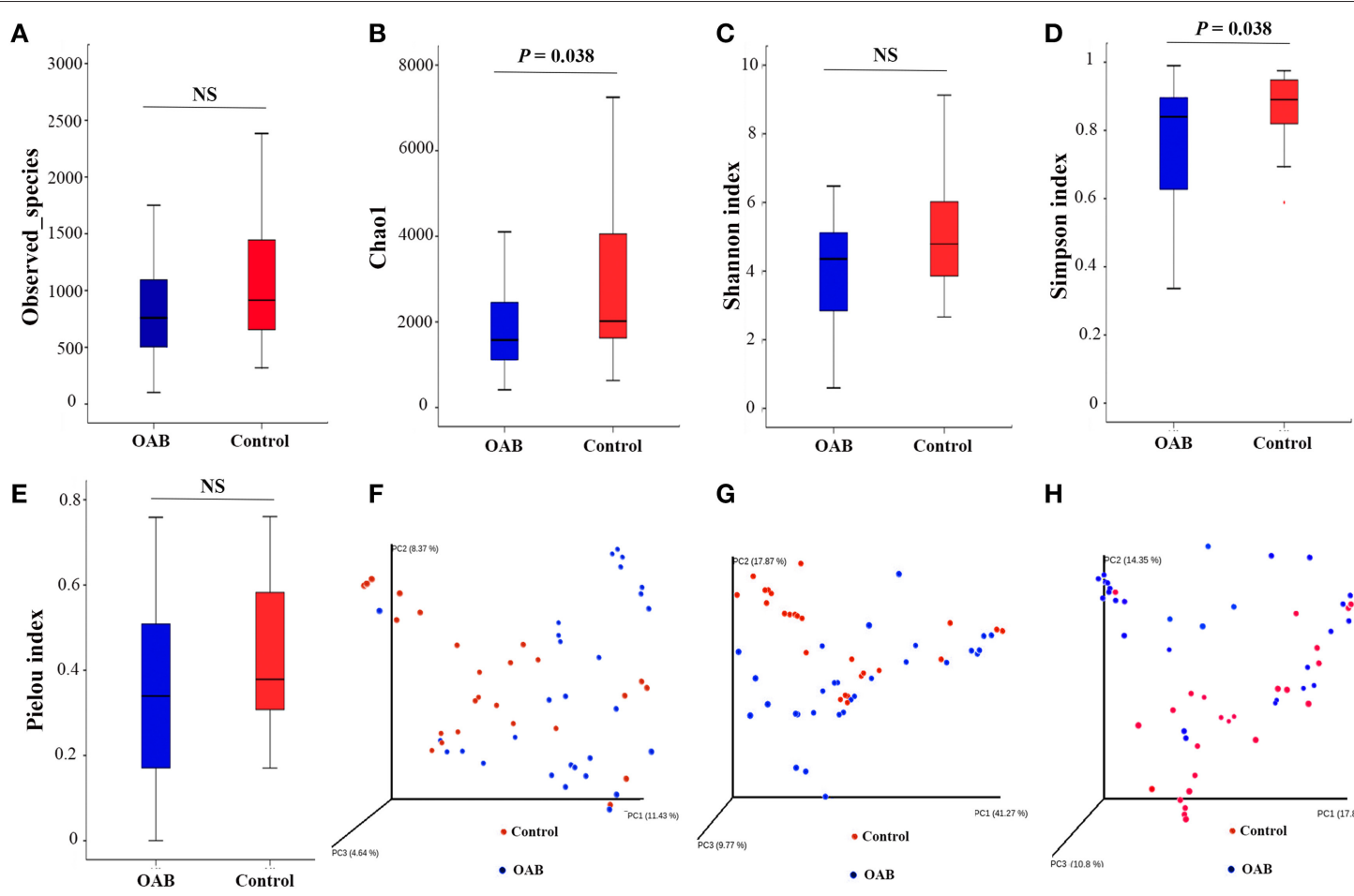

$\mathbf{F}$

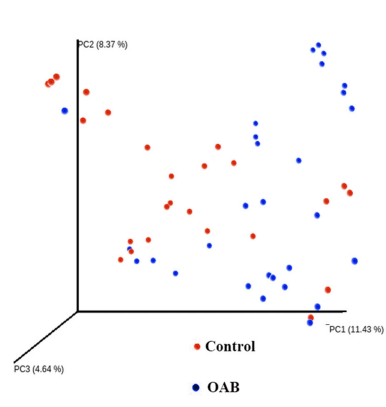

G

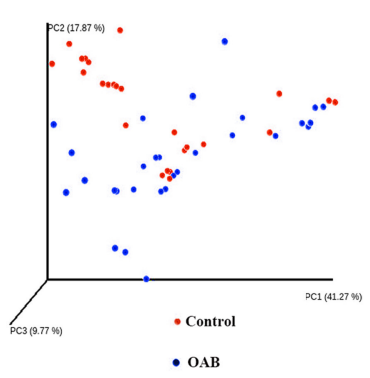

H

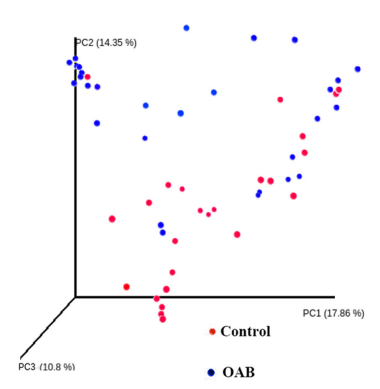

FIGURE 1 | Alpha diversity and principal coordinate analysis for control and OAB urinary microbiomes. Observed species (A); Chao1 (B); Shannon index (C); Simpson index (D); Pielou index (E). Principal coordinate analysis plot of the urinary microbiome based on the unweighted (F) or weighted (G) UniFrac and Bray-Curtis (H) distance metrics. 


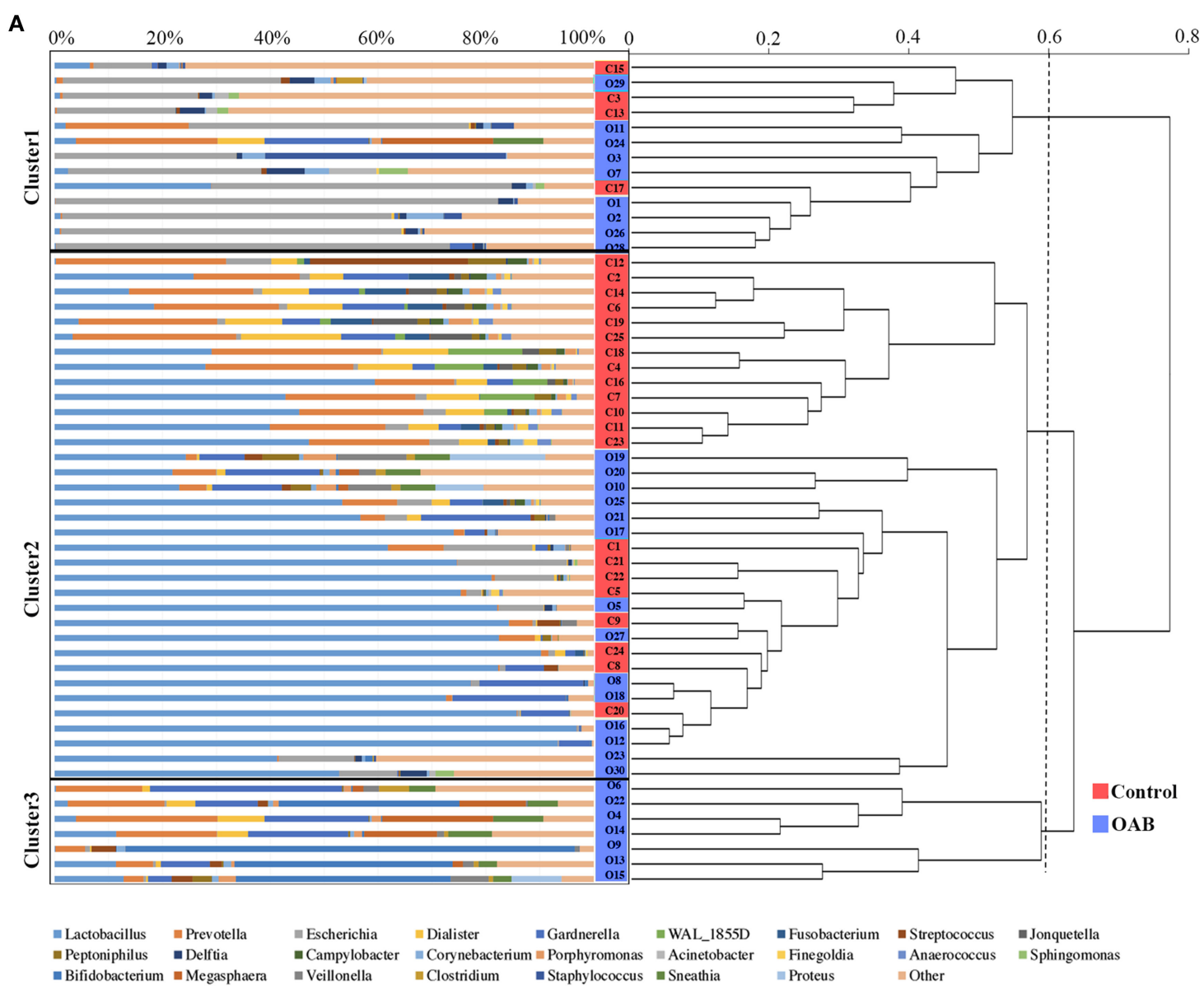

B

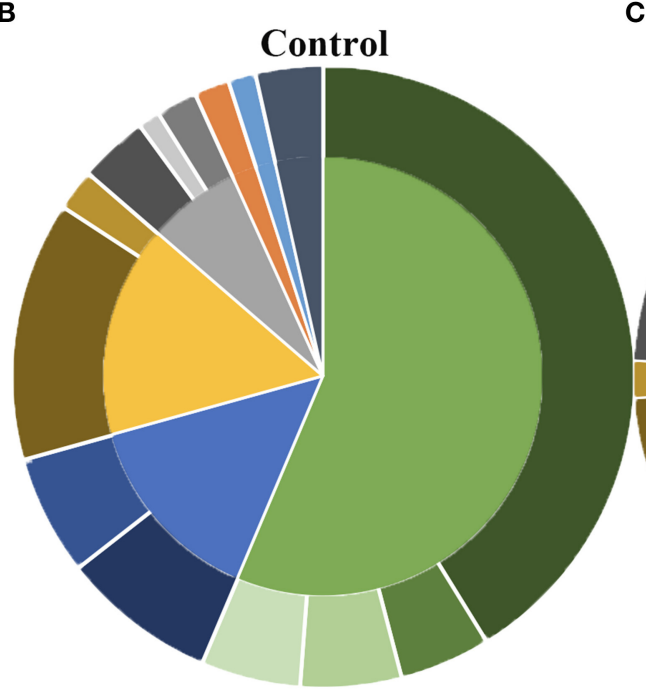

C

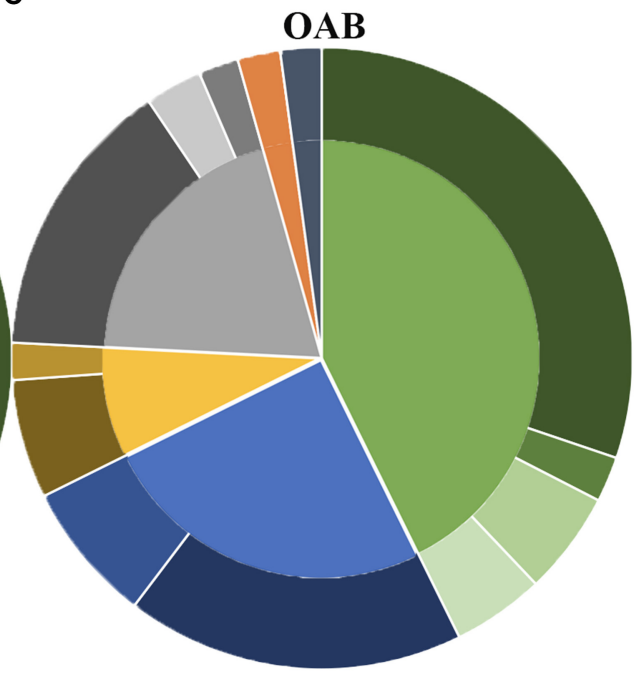

n Firmicutes

- Lactobacillaceae

- [Tissierellaceae]

" Veillonellaceae

- Other Firmicutes

- Proteobacteria

- Enterobacteriaceae

- Other Proteobacteria

Bacteroidetes

- Prevotellaceae

- Other Bacteroidetes

Actinobacteria

- Bifidobacteriaceae

$=$ Coriobacteriaceae

- Other Actinobacteria

- Fusobacteria

- Synergistetes

- Other

FIGURE 2 | The urinary microbiome profile of participants. The urinary microbiome profiles of the two cohorts cluster together, as showed in the dendrogram (right; based on the weighted UniFrac distance metric) and by the dominant genera present, as depicted in the histogram (left) (A). Comparison of taxonomic assignments for the controls $\mathbf{( B )}$ and OAB samples $\mathbf{( C )}$ at the Phyla level (inner circle) and family level (outer circle). Bacterial genera with a relative abundance $<0.5 \%$, are grouped as "Other." 

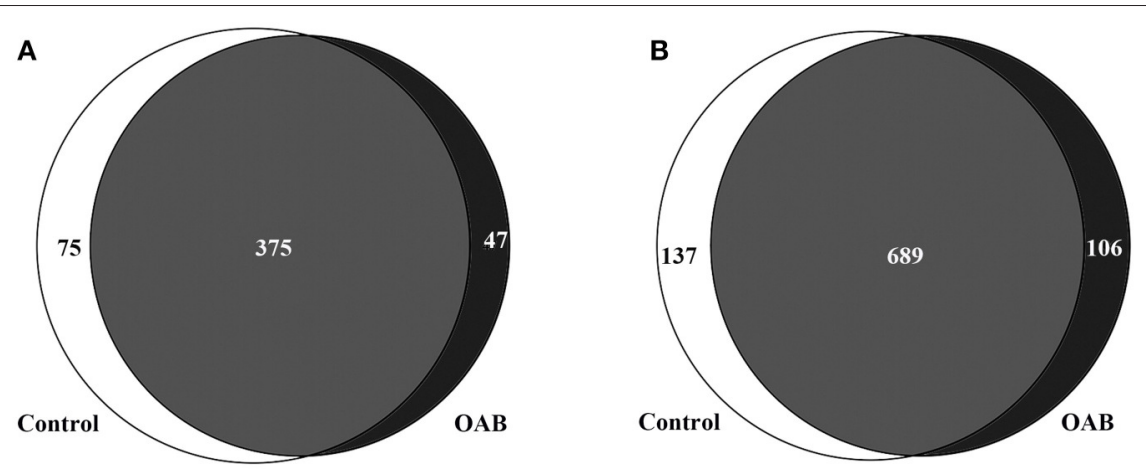

FIGURE 3 | Venn diagrams depicting the number of bacterial families (A) and genera (B) that are shared and unique between control and OAB urine.

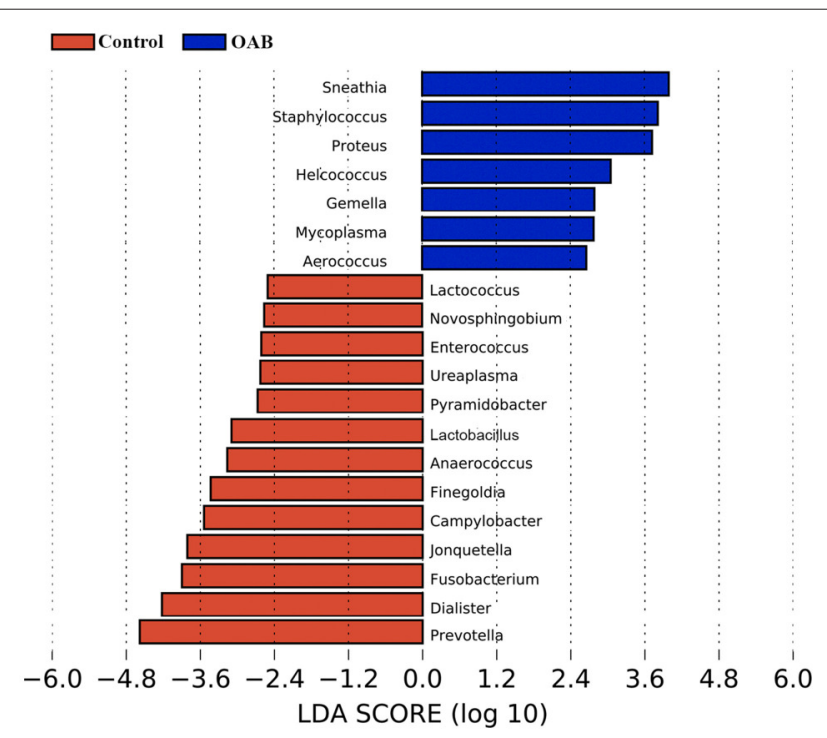

FIGURE 4 | LEfSe analyses of urinary microbiomes of OAB patients compared with controls. Genera enriched for controls in red; OAB patients enriched genera in blue. Only genera meeting a linear discriminant analysis score threshold $>2.5$ are shown.

urinary microbiome. Furthermore, 7 genera were increased and 13 were reduced compared to the control samples. We also found negative correlations between the depression score and both bacterial richness and diversity in the $\mathrm{OAB}$ patients, which inferred that psychological factors might play an important role in the urinary microbiome.

There have been some studies on the urinary microbiome of $\mathrm{OAB} / \mathrm{UUI}$. Hilt et al. identified bacteria associated with $\mathrm{OAB}$ by EQUC, and found that Aerococcus and Actinobaculum were isolated only from $\mathrm{OAB}$ urine (Hilt et al., 2014). Another study analyzing urinary microbiome collected from women with UUI and asymptomatic controls found that the microbiome from UUI displayed decreased Lactobacillus and increased Gardnerella abundance by sequence analysis, and nine genera were more frequently cultured from the UUI cohort by culture techniques (Pearce et al., 2014). Karstens et al. identified statistically significant differences in the relative abundance of specific bacteria in urine from women with UUI compared to controls using high throughput sequencing (Karstens et al., 2016). They also found that lower microbial diversity was associated with increased symptom severity in women with UUI. Our study shared some results with the above studies, such as increased genera (e.g., Aerococcus and Staphylococcus) and decreased genera (e.g., Prevotella and Lactobacillus) in the patient group. Hilt's group has evaluated the urinary microbiomes of hundreds of women with OAB/UUI and a smaller but substantial number of asymptomatic controls (Hilt et al., 2014; Pearce et al., 2014). It seems pretty clear that there are some bacterial species (including Aerococcus) associated with urinary symptoms and not with asymptomatic controls. However, there were several differences between these studies. An obvious difference was that Gardnerella, a genus representing one of the major urotypes in above previous studies, had low relative abundance in both cohorts in our study, similar to another study from China (Liu et al., 2017). Differences could be partly due to different clinical characteristics. Previous studies included populations with more severe urinary symptoms than our study. In addition, differences in reference databases used which can cause differences in the types of bacteria identified might also contribute to the discrepancies. Our study used the GreeneGenes database for taxanomic assignment, whereas other studies used the SILVA database. Another reason might be that the ethnic group in our study was different from that in the previous studies above. Notably, the gut microbiome was compositionally affected by geographical and ethnic factors (Prideaux et al., 2013). Future study focusing on whether there are differences in the urinary microbiomes between different racial types is needed.

The Chaol and Simpson index showed that the bacterial richness and diversity were lower in $\mathrm{OAB}$ samples compared to the asymptomatic samples. However, the bacterial evenness was not significantly different between the two groups, indicating that the shift in diversity was caused by the reduced richness. The urinary microbiome of asymptomatic women was found to be more diverse, and richer in Lactobacillus compared to the $\mathrm{OAB}$ group. A more diverse and Lactobacillus-dominated urinary microbiome might play a potentially protective role, 
A

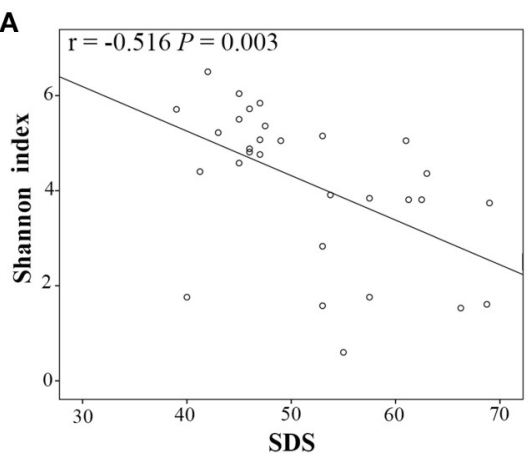

B

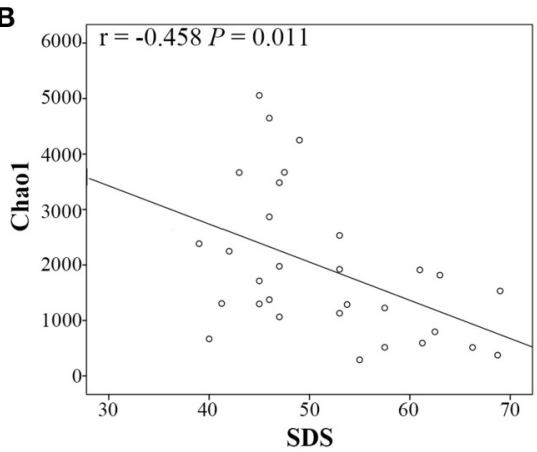

C

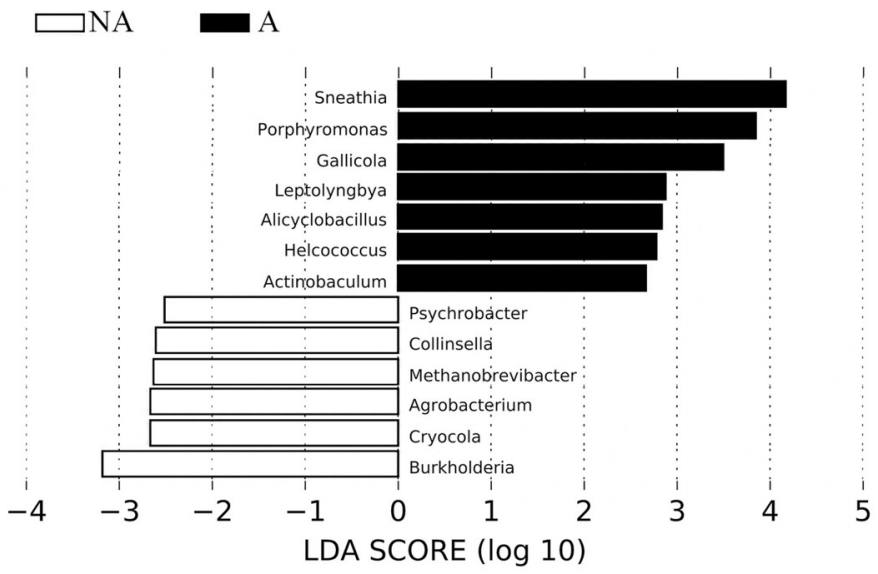

D $\square$ ND
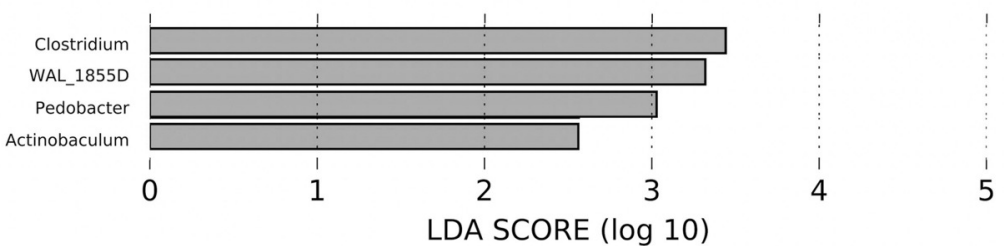

FIGURE 5 | Correlations between variables and significantly different bacterial genera between OAB patients with and without anxiety or depression. Higher SDS score inversely correlated with both Shannon index (A) and Chao1 (B). Bacterial genera differed significantly between OAB patients with and without anxiety based on LEfSe analyses (C). Bacterial genera differed significantly between OAB patients with and without depression based on LEfSe analyses (D). Only genera meeting a linear discriminant analysis score threshold $>2.5$ are shown. A, Anxiety; NA, non-anxiety; ND, non-depression; SDS, Self-rating Depression Scale.

which is consistent with arguments of other studies (Brubaker et al., 2014; Pearce et al., 2014). The profile of urinary microbiome in interstitial cystitis was also found to be less diverse and less likely to contain Lactobacillus species (Abernethy et al., 2017). This suggests that the intriguing possibility that specific microbial patterns may be linked to specific symptoms, regardless of diagnosis, which provides a great enlightenment for future researches of the urinary microbiome and the algorithms for the diagnosis and treatment of LUTS.

Although, we detected differences in several biologically plausible variables between the two groups, there was a great deal of individual variability among the $\mathrm{OAB}$ patients. As determined by $\mathrm{PCoA}$, there are overlapping regions where a subgroup of samples from $\mathrm{OAB}$ and controls are located. It suggests that some samples of $\mathrm{OAB}$ patients are very similar to controls, but some urinary microbiomes from $\mathrm{OAB}$ patients are quite distinct. Although, it was not statistically significant, the OAB patients whose urinary microbiomes were obviously different from the controls reported higher scores on OABSS, SAS, and SDS than in patients similar to controls. Moreover, different psychological conditions were associated with different severities of symptom and characteristics of urinary microbiome (Table 3 ). Similar to UUI (Pearce et al., 2014), this could be due to internal heterogeneity in the $\mathrm{OAB}$ population. The status of the urinary microbiome and mental factors should all be taken into consideration for clinical phenotyping of patients. The 
TABLE 3 | Comparisons of clinical symptoms and biodiversity of urinary microbiome between OAB patients with and without psychological disorders.

\begin{tabular}{|c|c|c|c|c|c|c|}
\hline & \multicolumn{2}{|c|}{ SAS } & \multirow[t]{2}{*}{$P$-value } & \multicolumn{2}{|c|}{ SDS } & \multirow[t]{2}{*}{$P$-value } \\
\hline & $<50(n=18)$ & $\geq 50(n=12)$ & & $<53(n=16)$ & $\geq 53(n=14)$ & \\
\hline SAS & $45.6(43.0,47.3)$ & $60.6(55.8,63.8)$ & $<0.001$ & $50.6(43.8,60.5)$ & $51.3(46.0,58.5)$ & 0.697 \\
\hline SDS & $48(41,55)$ & $51(46,62)$ & 0.305 & $45.0(42.0,47.0)$ & $58.5(52.5,63.0)$ & $<0.001$ \\
\hline OABSS & $6.9(5.0,8.3)$ & $9.1(8.0,10.0)$ & 0.007 & $7.0(6.0,8.0)$ & $8.6(7.5,10.0)$ & 0.043 \\
\hline Q1. Daytime frequency & $1.4(1.0,2.0)$ & $2.0(2.0,2.0)$ & 0.010 & $1.6(1.0,2.0)$ & $1.8(1.8,2.0)$ & 0.313 \\
\hline Q2. Nighttime frequency & $2.0(1.0,2.0)$ & $2.3(2.0,3.0)$ & 0.285 & $2.1(2.0,2.8)$ & $2.2(1.8,3.0)$ & 0.580 \\
\hline Q3. Urgency & $3.2(0,0)$ & $4.3(3.3,5.0)$ & 0.048 & $3.2(2.0,4.0)$ & $4.1(4.0,5.0)$ & 0.034 \\
\hline Q4. Urgency incontinence & $0.3(0,0)$ & $0.5(0,0)$ & 0.951 & $0.2(0,0)$ & $0.7(0,1.0)$ & 0.334 \\
\hline Observed species & $826.5(384.0,1136.0)$ & $785.0(515.5,1052.0)$ & 0.987 & 1,065.1 (782.8, 1297.3) & $518.3(307.5,679.8)$ & 0.002 \\
\hline Chao1 & $1,836.4(1014.3,2420.5)$ & 2,087.6 (862.8, 3465.8) & 0.723 & 2,604.3 (1322.5, 3668.5) & $1,174.1(514.8,1840.6)$ & $<0.001$ \\
\hline Shannon index & $4.0(2.7,5.4)$ & $4.5(3.8,5.2)$ & 0.662 & $5.1(4.8,5.7)$ & $3.1(1.6,4.0)$ & $<0.001$ \\
\hline Simpson index & $0.7(0.4,0.9)$ & $0.8(0.8,0.9)$ & 0.146 & $0.8(0.8,0.9)$ & $0.6(0.4,0.9)$ & 0.002 \\
\hline Pielou index & $0.3(0.2,0.4)$ & $0.5(0.3,0.6)$ & 0.217 & $0.3(0.2,0.5)$ & $0.4(0.2,0.6)$ & 0.423 \\
\hline
\end{tabular}

Data are reported as median (interquartile range). OABSS, Overactive Bladder Symptom Score; SDS, Self-Rating Depression Scale; SAS, Self-Rating Anxiety Scale.

careful phenotyping of patients gives a better understanding of heterogeneous pathology, and provides the basis for precise and personalized medicine, such as targeted therapy through removal of specific pathogens and the restoration of normal microbiome supplemented by psychotherapy.

Pathogenic organisms invading in urothelial cells were confirmed to be able to persist for a long time and serve as the primary source for bacterial expansion (Hunstad and Justice, 2010). Among the urine samples collected from chronic LUTS patients, $75 \%$ showed evidence of infected urothelial cells, which might stimulate the apoptotic process in urothelial cells and increase exposure of the underlying mucosal layer to irritative stimuli (Horsley et al., 2013). Low-count bacteriuria and intracellular bacterial communities were more prevalent among $\mathrm{OAB}$ women when a lower cut-off threshold $\left(10^{2} \mathrm{CFU} / \mathrm{mL}\right)$ was used (Khasriya et al., 2010). We identified 20 bacteria with significantly differential relative abundances between $\mathrm{OAB}$ microbiome and control microbiome. Of the 7 bacteria that had increased relative abundance in $\mathrm{OAB}$ urinary microbiome, some (Proteus, Staphylococcus, and Mycoplasma) are commonly seen in patients with UTI. This suggests that a persistent low grade infection by bacteria that are not commonly detected by standard culture could potentially be responsible for the OAB symptoms. It is a new dogma that the urine is not sterile and that many uropathogens are not detected by the standard culture method. The microbiome might be a new paradigm in urogenital infections and diseases, and influence the future classification of UTI.

There is an increasing understanding of the role of the braingut-microbiome axis in gastrointestinal diseases (Mayer et al., 2014). Evidence suggests that the axis is a complex bidirectional communication network between the gut microbiome and host, by which microbiome can modulate brain function, and conversely, chronic psychological stress may compound the degree of dysbiosis (Bailey et al., 2011). The urinary microbiome has similar potential. Interstitial cystitis was considered as a central sensitization syndrome with impaired inhibition of descending pain pathways (Ness et al., 2014). For idiopathic OAB patients, central sensitization may also help explain the pathogenesis (Reynolds et al., 2016). A study disclosed that different sets of bacterial taxa in urinary microbiome were increased in the psychosocial-predominant and neurologic-predominant groups of chronic pelvic pain syndrome (Shoskes et al., 2016). In our study, we identified significant correlations between measurements of depression severity and alpha diversity in women with OAB. The data indicates that $\mathrm{OAB}$ patients with depression have further reductions in bacterial diversity and richness. Furthermore, some bacterial genera differed significantly between $\mathrm{OAB}$ patients with and without anxiety or depression. It is an important finding that requires further studies to establish whether a brain-bladder-microbiome axis exists. It may become possible to manipulate brain status to regulate the urinary microbiome for purposes of treating urinary diseases, and vice versa, in the future.

This cross sectional study did contain several limitations that should be mentioned. First, we only measured the microbiome at one moment in time, thus it was difficult to determine the causeand-effect relationship between variables. Second, our study was limited by its small sample size. Further expansion of sample size is warranted to update our analysis and do a better comparison to existing studies.

Taken together, we examined differences in the urinary microbiomes of female $\mathrm{OAB}$ patients and asymptomatic controls, and then correlated microbiome with psychological conditions. The aberrant urinary microbiome may have strong implications in pathogenesis and treatment of OAB. Further studies are required to confirm the connection between urinary microbiome and central nervous system.

\section{AUTHOR CONTRIBUTIONS}

PW, YC, and JZ: conception and design; YC, GZ, and JC: acquisition of data; YC, JZ, and GZ: analysis and interpretation 
of data; PW, YC, and JZ: drafting of the manuscript; PW and HZ: critical revision of the manuscript for important intellectual content; PW, YC, JZ, and JW: statistical analysis; PW: obtaining funding; PW: supervision.

\section{FUNDING}

Supported by the Science and the Technology Planning Project of Guangdong Province (grant no. 2014A020212195), the

\section{REFERENCES}

Abernethy, M. G., Rosenfeld, A., White, J. R., Mueller, M. G., LewickyGaupp, C., and Kenton, K. (2017). Urinary microbiome and cytokine levels in women with interstitial cystitis. Obstet. Gynecol. 129, 500-506. doi: 10.1097/AOG.0000000000001892

Abrams, P., Cardozo, L., Fall, M., Griffiths, D., Rosier, P., Ulmsten, U., et al. (2002). The standardisation of terminology of lower urinary tract function: report from the standardisation sub-committee of the international continence society. Neurourol. Urodyn. 21, 167-178. doi: 10.1002/nau.10052

Bailey, M. T., Dowd, S. E., Galley, J. D., Hufnagle, A. R., Allen, R. G., and Lyte, M. (2011). Exposure to a social stressor alters the structure of the intestinal microbiota: implications for stressor-induced immunomodulation. Brain Behav. Immun. 25, 397-407. doi: 10.1016/j.bbi.2010.10.023

Bradley, C. S., Nygaard, I. E., Torner, J. C., Hillis, S. L., Johnson, S., and Sadler, A. G. (2014). Overactive bladder and mental health symptoms in recently deployed female veterans. J. Urol. 191, 1327-1332. doi: 10.1016/j.juro.2013.11.100

Brubaker, L., Nager, C. W., Richter, H. E., Visco, A., Nygaard, I., Barber, M. D., et al. (2014). Urinary bacteria in adult women with urgency urinary incontinence. Int. Urogynecol. J. 25, 1179-1184. doi: 10.1007/s00192-013-2325-2

Caporaso, J. G., Kuczynski,. J., Stombaugh, J., Bittinger, K., Bushman, F. D., Costello, E. K., et al. (2010). QIIME allows analysis of highthroughput community sequencing data. Nat. Methods 7, 335-336. doi: 10.1038/nmeth.f.303

Curtiss, N., Balachandran, A., Krska, L., Peppiatt-Wildman, C., Wildman, S., and Duckett, J. (2017). A case controlled study examining the bladder microbiome in women with Overactive Bladder (OAB) and healthy controls. Eur. J. Obstet. Gynecol. Reprod. Biol. 214, 31-35. doi: 10.1016/j.ejogrb.2017.04.040

Edgar, R. C. (2010). Search and clustering orders of magnitude faster than BLAST. Bioinformatics 26, 2460-2461. doi: 10.1093/bioinformatics/btq461

Fouts, D. E., Pieper, R., Szpakowski, S., Pohl, H., Knoblach, S., Suh, M. J., et al. (2012). Integrated next-generation sequencing of $16 \mathrm{~S}$ rDNA and metaproteomics differentiate the healthy urine microbiome from asymptomatic bacteriuria in neuropathic bladder associated with spinal cord injury. J. Transl. Med. 10:174. doi: 10.1186/1479-5876-10-174

Gormley, E. A., Lightner, D. J., Faraday, M., and Vasavada, S. P. (2015). American urological association, society of urodynamics, female pelvic medicine. diagnosis and treatment of overactive bladder (non-neurogenic) in adults: AUA/SUFU guideline amendment. J. Urol. 193, 1572-1580. doi: 10.1016/j.juro.2015.01.087

Hiergeist, A., and Gessner, A. (2017). Clinical implications of the microbiome in urinary tract diseases. Curr. Opin. Urol. 27, 93-98. doi: 10.1097/MOU.0000000000000367

Hilt, E. E., McKinley, K., Pearce, M. M., Rosenfeld, A. B., Zilliox, M. J., Mueller, E. R., et al. (2014). Urine is not sterile: use of enhanced urine culture techniques to detect resident bacterial flora in the adult female bladder. J. Clin. Microbiol. 52, 871-876. doi: 10.1128/JCM.02876-13

Horsley, H., Malone-Lee, J., Holland, D., Tuz, M., Hibbert, A., Kelsey, M., et al. (2013). Enterococcus faecalis subverts and invades the host urothelium in patients with chronic urinary tract infection. PLOS ONE 8:e83637. doi: 10.1371/journal.pone.0083637

Hunstad, D. A., and Justice, S. S. (2010). Intracellular lifestyles and immune evasion strategies of uropathogenic Escherichia coli. Annu. Rev. Microbiol. 64, 203-221. doi: 10.1146/annurev.micro.112408.134258
Natural Science Foundation of Guangdong Province (grant no. 2016A030313605), and the National Natural Science Foundation of China (Grant No.31401499).

\section{SUPPLEMENTARY MATERIAL}

The Supplementary Material for this article can be found online at: https://www.frontiersin.org/articles/10.3389/fcimb. 2017.00488/full\#supplementary-material

Karstens, L., Asquith, M., Davin, S., Stauffer, P., Fair, D., Gregory, W T., et al. (2016). Does the urinary microbiome play a role in urgency urinary incontinence and its severity? Front. Cell. Infect. Microbiol. 6:78. doi: $10.3389 /$ fcimb. 2016.00078

Khasriya, R., Khan, S., Lunawat, R., Bishara, S., Bignall, J., Malone-Lee, M., et al. (2010). The inadequacy of urinary dipstick and microscopy as surrogate markers of urinary tract infection in urological outpatients with lower urinary tract symptoms without acute frequency and dysuria. J. Urol. 183, 1843-1847. doi: 10.1016/j.juro.2010.01.008

Kinsey, D., Pretorius, S., Glover, L., and Alexander, T. (2016). The psychological impact of overactive bladder: a systematic review. J. Health Psychol. 21, 69-81. doi: 10.1177/1359105314522084

Lai, H. H., Shen, B., Rawal, A., and Vetter, J. (2016). The relationship between depression and overactive bladder/urinary incontinence symptoms in the clinical OAB population. BMC Urol. 16:60. doi: 10.1186/s12894-016-0179-x

Lewis, D. A., Brown, R., Williams, J., White, P., Jacobson, S. K., Marchesi, J. R., et al. (2013). The human urinary microbiome; bacterial DNA in voided urine of asymptomatic adults. Front. Cell. Infect. Microbiol. 3:41. doi: 10.3389/fcimb.2013.00041

Liu, F., Ling, Z., Xiao, Y., Lv, L., Yang, Q., Wang, B., et al. (2017). Dysbiosis of urinary microbiota is positively correlated with type 2 diabetes mellitus. Oncotarget 8, 3798-3810. doi: 10.18632/oncotarget.14028

Mayer, E. A., Knight, R., Mazmanian, S. K., Cryan, J. F., and Tillisch, K. (2014). Gut microbes and the brain: paradigm shift in neuroscience. J. Neurosci. 34, 15490-15496. doi: 10.1523/JNEUROSCI.3299-14.2014

Ness, T. J., Lloyd, L. K., and Fillingim, R. B. (2014). An Endogenous Pain Control System is altered in subjects with interstitial cystitis. J. Urol. 191, 364-370. doi: 10.1016/j.juro.2013.08.024

Nickel, J. C., Stephens, A., Landis, J. R., Chen, J., Mullins, C., van, Bokhoven, A., et al. (2015). Search for microorganisms in men with urologic chronic pelvic pain syndrome: a culture-independent analysis in the MAPP research network. J. Urol. 194, 127-135. doi: 10.1016/j.juro.2015.01.037

Pearce, M. M., Hilt, E. E., Rosenfeld, A. B., Zilliox, M. J., Thomas-White, K., Fok, C., et al. (2014). The female urinary microbiome: a comparison of women with and without urgency urinary incontinence. MBio. 5, e01283-e01214. doi: 10.1128/mBio.01283-14

Prideaux, L., Kang, S., Wagner, J., Buckley, M., Mahar, J. E., and De, Cruz, P., et al. (2013). Impact of ethnicity, geography, and disease on the microbiota in health and inflammatory bowel disease. Inflamm. Bowel Dis. 19, 2906-2918. doi: 10.1097/01.MIB.0000435759.05577.12

Reynolds, W. S., Dmochowski, R., Wein, A., and Bruehl, S. (2016). Does central sensitization help explain idiopathic overactive bladder? Nat. Rev. Urol. 13, 481-491. doi: 10.1038/nrurol.2016.95

Segata, N., Izard, J., Waldron, L., Gevers, D., Miropolsky, L., Garrett, W. S., et al. (2011). Metagenomic biomarker discovery and explanation. Genome Biol. 12:R60. doi: 10.1186/gb-2011-12-6-r60

Shoskes, D. A., Altemus, J., Polackwich, A. S., Tucky, B., Wang, H., and Eng, C. (2016). The urinary microbiome differs significantly between patients with chronic prostatitis/chronic pelvic pain syndrome and controls as well as between patients with different clinical phenotypes. Urology 92, 26-32. doi: 10.1016/j.urology.2016.02.043

Siddiqui, H., Lagesen, K., Nederbragt, A. J., Jeansson,. S. L., and Jakobsen, K. S. (2012). Alterations of microbiota in urine from women with interstitial cystitis. BMC Microbiol. 12:205. doi: 10.1186/1471-2180-12-205 
Stewart, W. F., Van Rooyen, J. B., Cundiff, G. W., Abrams, P., Herzog, A. R., Corey, R., et al. (2003). Prevalence and burden of overactive bladder in the United States. World J. Urol. 20, 327-336. doi: 10.1007/s00345-002-0301-4

Tadic, S. D., Griffiths, D., Schaefer, W., Cheng, C. I., and Resnick, N. M. (2010). Brain activity measured by functional magnetic resonance imaging is related to patient reported urgency urinary incontinence severity. J. Urol. 183, 221-228. doi: 10.1016/j.juro.2009.08.155

Thomas-White, K. J., Kliethermes, S., Rickey, L., Lukacz, E. S., Richter, H. E., Moalli, P., et al. (2017). Evaluation of the urinary microbiota of women with uncomplicated stress urinary incontinence. Am. J. Obstet. Gynecol. 216, 55.e1-55.e16. doi: 10.1016/j.ajog.2016.07.049

Wang, Q., Garrity, G. M., Tiedje, J. M., and Cole, J. R. (2007). Naive Bayesian classifier for rapid assignment of rRNA sequences into the new bacterial taxonomy. Appl. Environ. Microb. 73, 5261-5267. doi: 10.1128/AEM.00062-07

Whiteside, S. A., Razvi, H., Dave, S., Reid, G., and Burton, J. P. (2015). The microbiome of the urinary tract-a role beyond infection. Nat. Rev. Urol. 12, 81-90. doi: 10.1038/nrurol.2014.361
Wolfe, A. J., Toh, E., Shibata, N., Rong, R., Kenton, K., Fitzgerald, M., et al. (2012) Evidence of uncultivated bacteria in the adult female bladder. J. Clin. Microbiol. 50, 1376-1383. doi: 10.1128/JCM.05852-11

Young, V. B. (2017). The role of the microbiome in human health and disease: an introduction for clinicians. BMJ 356:j831. doi: 10.1136/bmj.j831

Conflict of Interest Statement: The authors declare that the research was conducted in the absence of any commercial or financial relationships that could be construed as a potential conflict of interest.

Copyright (c) 2017 Wu, Chen, Zhao, Zhang, Chen, Wang and Zhang. This is an open-access article distributed under the terms of the Creative Commons Attribution License (CC BY). The use, distribution or reproduction in other forums is permitted, provided the original author(s) or licensor are credited and that the original publication in this journal is cited, in accordance with accepted academic practice. No use, distribution or reproduction is permitted which does not comply with these terms. 